\title{
Numerical Study of Nonperturbative Corrections to the Chiral Separation Effect in Quenched Finite-Density QCD
}

\author{
Matthias Puhr ${ }^{*}$ and P. V. Buividovich ${ }^{\dagger}$ \\ Institute of Theoretical Physics, Regensburg University, 93040 Regensburg, Germany \\ (Received 1 December 2016; revised manuscript received 30 March 2017; published 10 May 2017) \\ We demonstrate the nonrenormalization of the chiral separation effect (CSE) in quenched finite-density \\ QCD in both confinement and deconfinement phases using a recently developed numerical method which \\ allows us, for the first time, to address the transport properties of exactly chiral, dense lattice fermions. This \\ finding suggests that CSE can be used to fix renormalization constants for axial current density. Explaining \\ the suppression of the CSE which we observe for topologically nontrivial gauge field configurations on \\ small lattices, we also argue that CSE vanishes for self-dual non-Abelian fields inside instanton cores.
}

DOI: 10.1103/PhysRevLett.118.192003

Anomalous transport phenomena which involve collective motion of chiral fermions are important in many disparate subfields of physics ranging from cosmology and astrophysics [1-4] over solid-state physics [5-7] to high energy physics and heavy-ion collision experiments [8-11]. Well-known examples of such phenomena are the induction of vector or axial currents along the magnetic field in a dense chiral medium, dubbed the chiral magnetic effect (CME) [8,12] and the chiral separation (CSE) effect $[3,8,13,14]$, respectively. In particular, for quark-gluon plasma produced in off-central heavy-ion collisions, CSE can locally induce large chirality imbalance [15], and, combined with CME, lead to a novel gapless hydrodynamic excitation-the chiral magnetic wave [16].

Within the hydrodynamic approximation, the requirement of positive entropy production together with the Adler-Bell-Jackiw axial anomaly equation fix the transport coefficients describing CME and CSE $[17,18]$. However, the hydrodynamic approximation used in Refs. $[17,18]$ might become invalid if the chiral plasma features an infinite correlation length (e.g., due to spontaneous symmetry breaking [19]) or interacts with dynamical Yang-Mills fields [20]. This allows for nonperturbative corrections to CME and CSE. Interactions with dynamic electromagnetic fields also lead to perturbative corrections $[21,22]$, which we do not consider in this work.

For CSE in QCD matter, which is in the focus of this work, the nonperturbative correction can be expressed in terms of the in-medium amplitude $g_{\pi^{0} \gamma \gamma}$ of the $\pi^{0} \rightarrow \gamma \gamma$ decay [14]:

$$
j_{i}^{5}=\sigma_{\mathrm{CSE}} B_{i}, \quad \sigma_{\mathrm{CSE}}=\sigma_{\mathrm{CSE}}^{0}\left(1-g_{\pi^{0} \gamma \gamma}\right),
$$

where $j_{i}^{5}$ is the axial current density and $B_{i}$ is the external magnetic field. With $g_{\pi^{0} \gamma \gamma}=0$, we recover the result $\sigma_{\mathrm{CSE}}^{0}=q N_{c} \mu / 2 \pi^{2}$ for $N_{c}$ species of free chiral fermions (with $N_{c}$ being the number of colors), which is also expected to be valid in the high-temperature phase with restored chiral symmetry $[3,14,23]$.
Within the linear sigma model, an estimate of $g_{\pi^{0} \gamma \gamma}$ for a medium with spontaneously broken chiral symmetry and at sufficiently small quark chemical potential $\mu$ is $g_{\pi^{0} \gamma \gamma}=7 \zeta(3) m^{2} / 4 \pi^{2} T^{2}$, where $\zeta$ is the Riemann $\zeta$ function, $m$ is the constituent quark mass, and $T$ is the temperature [14]. With realistic values $m \sim 300 \mathrm{MeV}$ [24] and $T \sim 150 \mathrm{MeV}$, which provide a reasonably good description of the chirally broken phase, we get a correction of order of $100 \%$, which suppresses the CSE response. Nonperturbative corrections which suppress CSE were also predicted within the Nambu-Jona-Lasinio model [25-27] and within the holographic model of a chiral superfluid with broken Abelian global symmetry [28,29].

Since nonperturbative corrections to anomalous transport phenomena might significantly modify the predictions of anomalous hydrodynamics, it is important to quantify them in a model-independent way in first-principles lattice QCD simulations. Thus far, a few lattice studies have addressed the infrared values of anomalous transport coefficients characterizing the CME $[30,31]$ and the chiral vortical effect $[32,33]$ and found a very significant suppression of the CME and chiral vortical effect at both low and high temperatures. This is a very puzzling situation, since at least at high temperatures one can expect that the thermodynamic consistency arguments [17,18] fixing anomalous transport coefficients in hydrodynamic approximation should be valid. Possible reasons for this discrepancy might be the use of naively discretized, nonconserved vector current [30,31] and energy-momentum tensor [32,33], and the use of nonchiral Wilson-Dirac fermions in Refs. [30,31]. In summary, this situation clearly calls for more accurate first-principles studies of anomalous transport coefficients which would be free of systematic errors.

In this Letter we report on a first-principles lattice study of CSE with finite-density overlap fermions [34], which respect the lattice chiral symmetry at any chemical potential. We use the properly defined lattice counterpart of the continuum axial current density $j_{\mu}^{5}=\bar{\psi} \gamma_{5} \gamma_{\mu} \psi[35,36]$ : 


$$
j_{x, \mu}^{5}=\frac{1}{2} \bar{\psi}\left[-\gamma_{5} K_{x, \mu}+K_{x, \mu} \gamma_{5}\left(1-D_{\mathrm{ov}}\right)\right] \psi,
$$

where $K_{x, \mu}=\partial D_{\text {ov }} / \partial \Theta_{x, \mu}$ is the derivative of the overlap operator $D_{\text {ov }}$ over the $U(1)$ lattice gauge field $\Theta_{x, \mu}$. The lattice axial current Eq. (2) transforms covariantly under the lattice chiral symmetry, and is hence protected from renormalization at zero quark mass and can be directly related to the continuum axial current in Eq. (1). After some algebra, taking the expectation value on both sides of Eq. (2) yields

$$
\left\langle j_{x, \mu}^{5}\right\rangle=\operatorname{tr}\left(D_{\mathrm{ov}}{ }^{-1} \frac{\partial D_{\mathrm{ov}}}{\partial \Theta_{x, \mu}} \gamma_{5}\right) .
$$

Technically, the most advanced problem is the calculation of the derivatives $\partial D_{\text {ov }} / \partial \Theta_{x, \mu}$ which enter the definitions of conserved vector and axial currents for overlap fermions. To this end we have developed a special algorithm, described in a separate paper [37].

Lattice QCD with dynamical fermions suffers from a fermionic sign problem at finite quark chemical potential. Moreover, a sign problem seems to be in general unavoidable for gauge theories with dense fermions in a magnetic field, since an external magnetic field breaks time-reversal and/or charge-conjugation symmetries which otherwise ensure the positivity of path integral weight for gauge theories with isospin chemical potential or $S U(2)$ or $G_{2}$ gauge groups. To avoid the fermionic sign problem, in the present work we neglect the effect of sea quarks and work in the quenched approximation, which was also used for holographic studies of CSE [28,29]. While arguments from a QCD random matrix model suggest that in the quenched approximation any nonzero chemical potential leads to a vanishing chiral condensate and thus restores chiral symmetry [38], the situation might be different for a magnetized QCD matter, where random matrix theory becomes inapplicable, and nonperturbative corrections to CSE appear due to spontaneous generation of the so-called chiral shift parameter [25-27], rather than chiral condensate.

The $S U(3)$ gauge configurations for our calculations were generated using the tadpole-improved Lüscher-Weisz gauge action [39]. We chose three different lattice setups: $V=L_{T} \times$ $L_{S}{ }^{3}=6 \times 18^{3}$, with $\beta=8.45$ corresponding to a temperature $T>T_{c}$, and $V=14 \times 14^{3}$ and $V=8 \times 8^{3}$, with $\beta=$ 8.10 corresponding to $T<T_{c}$, where $L_{T}$ and $L_{S}$ are the temporal and spatial extent of the lattice and $T_{c} \approx 300 \mathrm{MeV}$ is the deconfinement transition temperature of the LüscherWeisz action [40]. The physical value of the lattice spacing $a$ was determined using results from Ref. [41].

For the $14 \times 14^{3}$ and $6 \times 18^{3}$ lattices we generated around $10^{3}$ gauge configurations, from which we randomly picked 100 configurations with topological charge $Q=0$ [42]. For $6 \times 18^{3}$ we also chose 100 configurations with topological charge $|Q|=1$, and for $14 \times 14^{3} 111$ with
$|Q|=1$, and 97 with $|Q|=2$. For the $8 \times 8^{3}$ lattice we generated $5 \times 10^{3}$ configurations, from which three random sets of 200 configurations with $Q=0,|Q|=1$, and $|Q|=2$ were selected. We calculated the absolute value of topological charge $|Q|=\left|n_{R}-n_{L}\right|$ as the number of zero eigenvalues of the operator $D_{\mathrm{ov}} D_{\mathrm{ov}}^{\dagger}$, relying on the fact that, in practice, the overlap operator always has either $n_{R}=|Q|$ right-handed or $n_{L}=|Q|$ left-handed zero modes (see, e.g., Sec. VII. C. 2 in Ref. [43]).

We further introduced a constant, homogeneous external magnetic field following the prescription of Ref. [44] with magnetic flux quantum $\Phi_{B}=1,2,5,10$ for $V=14 \times 14^{3}$ and $V=6 \times 18^{3}$ at $Q=0$, and $\Phi_{B}=0,1,2,3,4$ for $V=8 \times 8^{3}$ at all $Q$. For $V=6 \times 18^{3}$ we chose $\Phi_{B}=0,1$, 2, 3, 5 at $|Q|=1$ and $\Phi_{B}=1,3,5,8,10$ for $V=14 \times 14^{3}$ at $|Q|=1,2$. For each parameter set, we evaluated the axial current density averaged over the lattice volume for one or two different values of the quark chemical potential $\mu$. The trace in Eq. (2) was calculated using stochastic estimators with $Z_{2}$ noise. We increased the number of stochastic estimators until the results were stable [see Figs. 1(a) and 1 (b) for confidence intervals on $\sigma_{\mathrm{CSE}}$ with different numbers of estimators]. For configurations with nonzero topological charge, we introduced a small quark mass $m_{q}=0.001 a^{-1}$ to make the Dirac operator invertible. To demonstrate that finite quark mass has practically no effect on $\sigma_{\mathrm{CSE}}$, for the $8 \times 8^{3}$ lattice we also considered another value $m_{q}=0.002 a^{-1}$. Our simulation parameters are summarized in Table I.

The value of $\sigma_{\mathrm{CSE}}$ is given by the slope of the axial current density as a function of the external magnetic field and can be found by performing a one parameter linear fit to the axial current data (the offset is fixed to zero, since the current has to vanish for $B=0$ ). Confidence intervals for $\sigma_{\mathrm{CSE}}$ were computed with the statistical bootstrap, by independently drawing bootstrap samples for every value of $\Phi_{B}$ and fitting the data generated in this way.

First, we consider the high-temperature deconfinement phase with $T=346 \mathrm{MeV}>T_{c}$, where chiral symmetry should be at least partially restored as compared to the confinement phase (see, e.g., Refs. [45,46] for a discussion of chiral symmetry restoration in quenched QCD). In this case, CSE is expected to have no corrections to the free fermion result $[3,14,23]$; i.e., $g_{\pi^{0} \gamma \gamma}\left(T>T_{c}\right)=0$. To check this expectation, in Fig. 1(a) we plot our results for the axial current density for configurations with zero topological charge as a function of $q B$ (data points with error bars). Shaded regions show the bootstrap confidence intervals for different numbers of stochastic estimators which lie on top of each other; hence, the error cannot be improved by using more estimators in the trace calculation. We find in general a good agreement with the free fermion result $\sigma_{\mathrm{CSE}}^{0}$, except for the largest values $\Phi_{B}=10$ and $\mu=0.230 a^{-1}$, for which we might see some saturation effect. Therefore, we also perform separate fits excluding the data for 


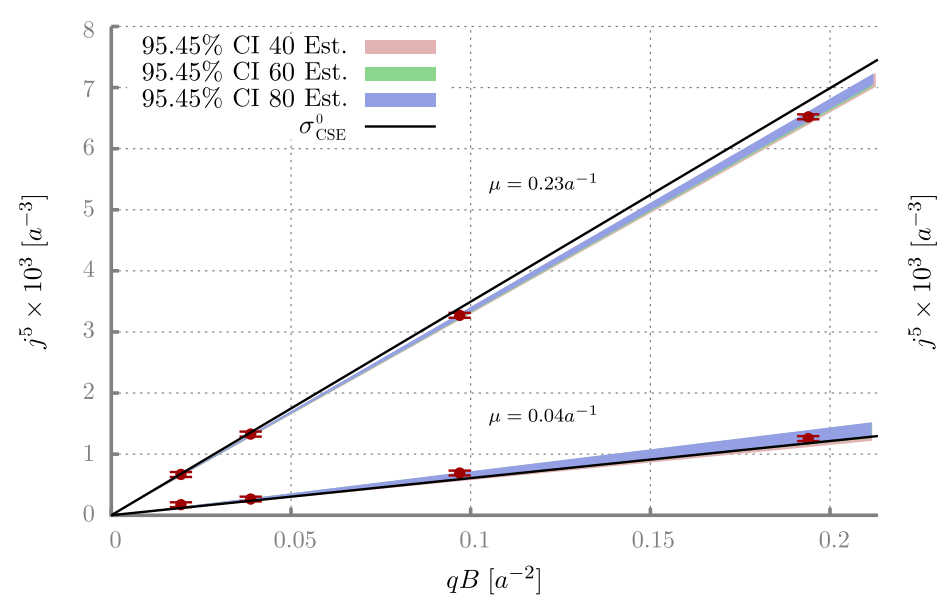

(a) $T>T_{c}, V=6 \times 18^{3}$

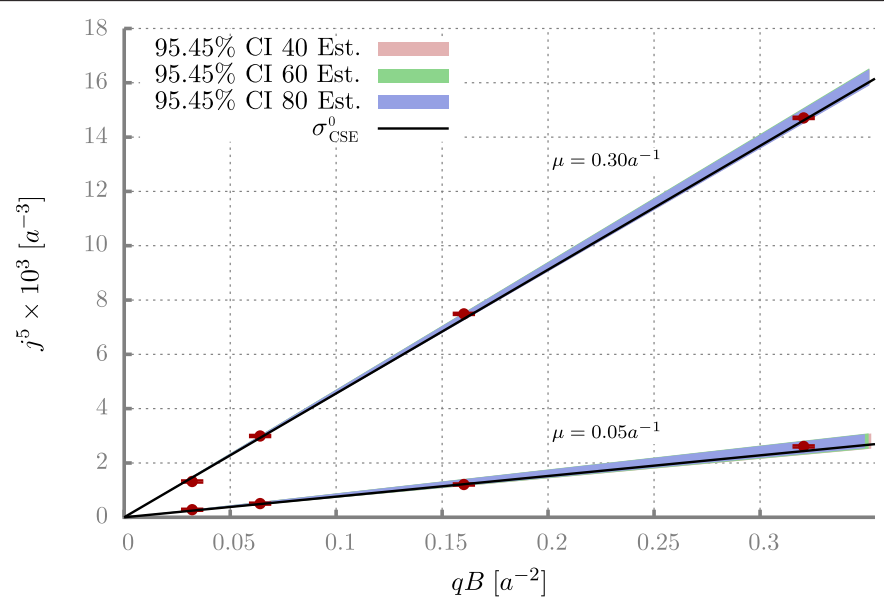

(b) $T<T_{c}, V=14 \times 14^{3}$

FIG. 1. Axial current density as a function of the magnetic field strength for topological charge $Q=0$ (red circles with error bars) at $T>T_{c}$ (left) and $T<T_{c}$ (right). Black lines correspond to the free fermion result $\sigma_{\mathrm{CSE}}^{0}$ for both values of the chemical potential. The shaded regions mark the confidence intervals (CI) for $\sigma_{\mathrm{CSE}}$ with different numbers of stochastic estimators.

$\Phi_{B}=10$, which show much better agreement with $\sigma_{\mathrm{CSE}}^{0}$. For the larger chemical potential value the signal-to-noise ratio is very good and the relative error of the slope measurement is smaller than $10 \%$. For configurations with $|Q|=1$, we also find a good agreement with $\sigma_{\mathrm{CSE}}^{0}$ within statistical errors. The results for the confidence intervals of $\sigma_{\mathrm{CSE}}$ are summarized in Fig. 2. We conclude that within our statistical errors corrections to CSE are absent in the deconfinement phase of quenched QCD.

We now consider the low-temperature confinement phase at $T<T_{c}$, where nonperturbative corrections to CSE can be expected [25-29]. In Fig. 1(b), we plot the axial current density as a function of the magnetic field strength for gauge field configurations with zero topological charge on the $14 \times 14^{3}$ lattice with $T=113 \mathrm{MeV}$ and $m_{q}=0$. The composition of the plot is the same as for Fig. 1(a). The confidence intervals for $\sigma_{\mathrm{CSE}}$ are very small and, again, contain the free fermion result within statistical errors. For the best fits at large chemical potential, the

TABLE I. Simulation parameters.

\begin{tabular}{lcccc}
\hline \hline \multirow{2}{*}{ Setup } & $\beta$ & 8.1 & 8.1 & 8.45 \\
& Volume & $14 \times 14^{3}$ & $8 \times 8^{3}$ & $6 \times 18^{3}$ \\
\hline$a(\mathrm{fm})$ & Lattice & \multicolumn{3}{c}{ Physical value } \\
$V_{S}\left(\mathrm{fm}^{3}\right)$ & 1 & 0.125 & 0.125 & 0.095 \\
$T(\mathrm{MeV})$ & $L_{S}{ }^{3}$ & 5.4 & 1.0 & 5.0 \\
$\mu(\mathrm{MeV})$ & $L_{T}^{-1}$ & 113 & 197 & 346 \\
& 0.050 & 79 & $\ldots$ & $\ldots$ \\
& 0.100 & $\ldots$ & 158 & $\ldots$ \\
& 0.300 & 474 & $\ldots$ & $\ldots$ \\
& 0.040 & $\ldots$ & $\ldots$ & 83 \\
$q B / \Phi_{B}(\mathrm{MeV})^{2}$ & 0.230 & $\ldots$ & $\ldots$ & 478 \\
\hline \hline
\end{tabular}

relative error of the slope is smaller than $6 \%$. Even for the highest magnetic field strength and the largest chemical potential, we do not see any saturation of the axial current. For configurations with $|Q|=1$, we again find that $\sigma_{\mathrm{CSE}}$ agrees with the free fermion result $\sigma_{\mathrm{CSE}}^{0}$ within confidence intervals (see Figs. 2 and 3). We thus conclude that even in the low-temperature phase of quenched QCD, the CSE does not receive any nonperturbative corrections.

At an early stage of this work, we also performed calculations with small lattice volume $V=8 \times 8^{3}$ at $\beta=$ 8.1 and $\mu=0.1 a^{-1}=158 \mathrm{MeV}$ (see also Table I). While in the zero topological sector we found $\sigma_{\mathrm{CSE}}$ to agree with the free fermion result $\sigma_{\mathrm{CSE}}^{0}$ within statistical errors, which indicates the smallness of finite-volume effects in the $Q=0$ sector, for

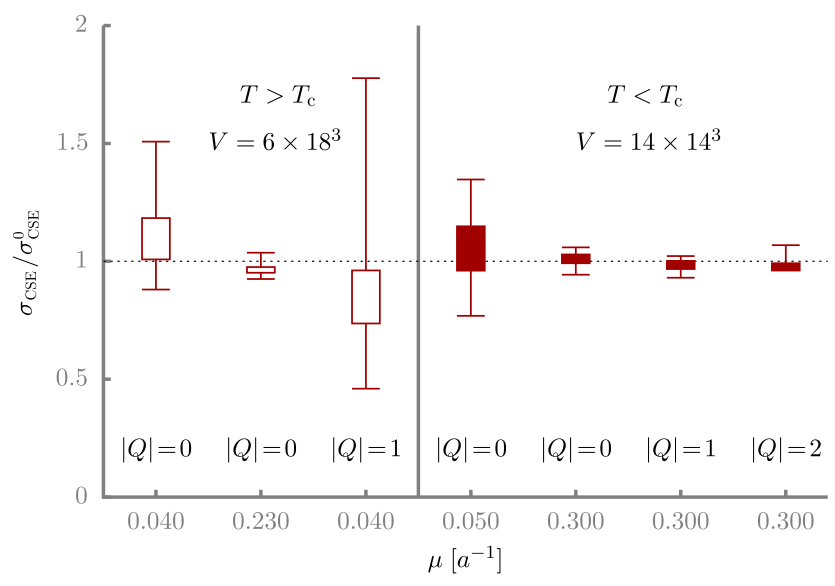

FIG. 2. Confidence intervals for the ratio $\sigma_{\mathrm{CSE}} / \sigma_{\mathrm{CSE}}^{0}$ for different values of the chemical potential and the topological charge. Boxes and whiskers mark the confidence interval for a fit with all data points and with the largest value of $\Phi_{B}$ excluded, respectively. Filled and open boxes are the results for $T<T_{c}$ and $T>T_{c}$, respectively. 


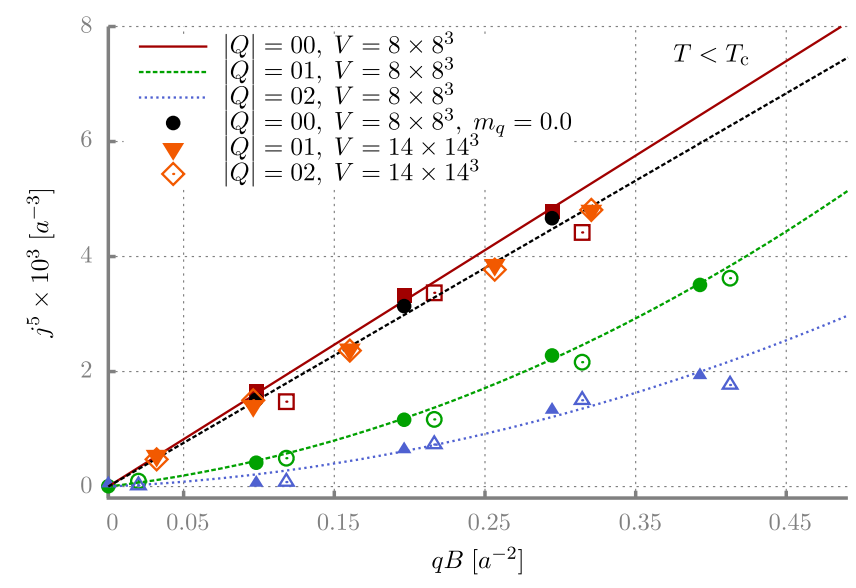

FIG. 3. The axial current in different topological sectors. Filled symbols mark the results for $m_{q}=0.001 a^{-1}$; the results for $m_{q}=$ $0.002 a^{-1}$ are shifted by $0.02 a^{-2}$ in the $q B$ axis for better visibility and are marked by open symbols. The black dots denote the axial current with $Q=0$ for vanishing quark mass, and the black dashed line corresponds to the free fermion result $\sigma_{\mathrm{CSE}}^{0}$. To guide the eye a linear $(Q=0)$ or second order polynomial $(|Q|>0)$ fit to the data is shown. For comparison, we also plot the results for $|Q|>0$ for the $14 \times 14^{3}$ lattice.

configurations with nonzero topological charge we found a rather strong suppression of CSE as well as a nonlinear dependence of the axial current on the magnetic field, as illustrated in Fig. 3. We checked that these findings are not finite mass effects by doing calculations with two masses $m_{q}=$ $0.001 a^{-1}=1.6 \mathrm{MeV}$ and $m_{q}=0.002 a^{-1}=3.2 \mathrm{MeV}$, which yield almost identical results. Furthermore, we found that the negative contribution to the axial current which suppresses the CSE comes exclusively from topological modes of the Dirac operator (the eigenvectors which would correspond to zero eigenvalues of the Dirac operator with $m_{q}=0$ ).

In order to understand the possible origin of the suppression of CSE on topologically nontrivial configurations in small volume, let us consider CSE in a background of constant Euclidean self-dual non-Abelian gauge field, which can be interpreted as a limiting case of very large instanton [47]. For gauge configurations with nonzero topological charge on a small lattice, this is a reasonable approximation, as in most cases only a few instantons would fit in a finite box with physical size $L=8 a=1.0 \mathrm{fm}$ comparable with the characteristic instanton size [48]. In the gauge where the timelike component of the vector gauge potential depends only on the longitudinal spatial coordinate, thus giving rise to a constant chromoelectric field, eigenstates of the Dirac operator can be labeled by the timelike momentum $k_{0}$. Introducing a finite chemical potential leads to a shift $k_{0} \rightarrow k_{0}-i \mu$. However, due to relativistic Landau quantization in a Euclidean electric field, the dependence of Dirac operator eigenvectors on $k_{0}$ reduces to global spatial shifts along the electric field [44,47], and the corresponding eigenvalues do not depend on $k_{0}$. Upon analytic continuation to complex values $k_{0} \rightarrow k_{0}-i \mu$, this $k_{0}$ independence translates into the independence of volume-averaged axial and vector currents on the chemical potential. Since at zero $\mu$ the axial current vanishes, it also vanishes at finite $\mu$ [49]. This argument suggests a "porous" spatial distribution of axial current induced due to CSE, which should vanish in regions with self-dual gauge fields. Within the instanton liquid model, these regions can be identified with instanton cores.

To conclude, our numerical study suggests that nonperturbative corrections to the chiral separation effect are either very small (smaller than our statistical errors) or vanishing for finite-density quenched QCD in the thermodynamic limit. By using finite-density overlap fermions [50] with covariant axial current, we have eliminated systematic errors due to explicit breaking of chiral symmetry and axial current renormalization. Finite-volume effects also seem to be rather small at least in the zero topology sector. Thus, the quenched approximation seems to be the only potentially important source of systematic errors. Indeed, one might argue that we do not find any nonperturbative corrections predicted in Ref. [14], since quenched QCD at any nonzero chemical potential is in the chirally symmetric phase with zero chiral condensate [38]. However, the arguments of Ref. [38], which are based on a random matrix model of QCD, might not be directly applicable to QCD in sufficiently strong external magnetic fields, which should introduce certain correlations in the otherwise statistically independent entries of the random matrix which mimics the QCD Dirac operator. Furthermore, calculations within the Nambu-Jona-Lasinio model [25-27] suggest that nonperturbative corrections to CSE are related to spontaneous generation of the so-called chiral shift parameter, which, in contrast to chiral condensate, cannot be described within the random matrix model framework of Ref. [38]. Finally, let us recall that also the holographic calculations [28,29], which do predict nonperturbative corrections to CSE at low temperatures, were performed in the quenched approximation ("probe limit" in AdS/QCD terminology).

Of course, these arguments simply illustrate that the nonrenormalization of CSE in quenched QCD at both high and low temperatures is a nontrivial result. They do not prohibit nonperturbative corrections that might originate, for example, from the complex phase which the fermion determinant acquires at finite density. Note that the external magnetic field renders the fermion determinant complex valued even for $S U(2)$ or $G_{2}$ gauge theories which are otherwise free of the sign problem. Since in massless QCD strong oscillations of the determinant phase related to the "silver blaze" phenomenon can be expected to set in already at very small density [51,52], the study of CSE in full QCD with dynamical fermions might be technically very challenging and is out of the scope of this work.

The nonrenormalization of CSE at least in quenched QCD can also have a practical application to the calculation 
of the renormalization constant for the axial current. Namely, the ratio of the CSE-induced axial current calculated with nonchiral lattice fermions and/or some noncovariant discretization of the axial current to the exact result $j_{i}^{5}=\left(\mu / 2 \pi^{2}\right) B_{i}$ yields the multiplicative renormalization constant for the axial current density in this particular discretization.

Finally, we note that the precision with which our results reproduce the theoretically expected value $\sigma_{\mathrm{CSE}}=\sigma_{\mathrm{CSE}}^{0}$ demonstrates that the approach to finite-density overlap fermions developed in Ref. [50] and further in Ref. [37] provides a reliable tool for first-principles numerical studies of transport properties of dense chiral fermions in lattice QCD.

This work was supported by the S. Kowalevskaja award from the Alexander von Humboldt Foundation. The calculations were performed on "iDataCool" at Regensburg University, on the ITEP cluster in Moscow, and on the LRZ cluster in Garching. We acknowledge valuable discussions with G. Bali, A. Dromard, and A. Zhitnitsky. M. P. thanks Rudolf Rödl for helpful comments on the statistical bootstrap.

*Matthias.Puhr@physik.uni-regensburg.de

${ }^{\dagger}$ Pavel.Buividovich@physik.uni-regensburg.de

[1] A. Vilenkin, Phys. Rev. D 22, 3080 (1980).

[2] H. Tashiro, T. Vachaspati, and A. Vilenkin, Phys. Rev. D 86, 105033 (2012).

[3] M. A. Metlitski and A. R. Zhitnitsky, Phys. Rev. D 72, 045011 (2005).

[4] G. Sigl and N. Leite, J. Cosmol. Astropart. Phys. 01 (2016) 025.

[5] H. J. Kim, K. S. Kim, J. F. Wang, M. Sasaki, N. Satoh, A. Ohnishi, M. Kitaura, M. Yang, and L. Li, Phys. Rev. Lett. 111, 246603 (2013).

[6] Q. Li, D. E. Kharzeev, C. Zhang, Y. Huang, I. Pletikosic, A. V. Fedorov, R. D. Zhong, J. A. Schneeloch, G. D. Gu, and T. Valla, Nat. Phys. 12, 550 (2016).

[7] G. Basar, D. E. Kharzeev, and H. U. Yee, Phys. Rev. B 89, 035142 (2014).

[8] D. Kharzeev and A. Zhitnitsky, Nucl. Phys. A797, 67 (2007).

[9] D. E. Kharzeev, L. D. McLerran, and H. J. Warringa, Nucl. Phys. A803, 227 (2008).

[10] L. Adamczyk (STAR Collaboration), Phys. Rev. Lett. 113, 052302 (2014).

[11] D. E. Kharzeev, J. Liao, S. A. Voloshin, and G. Wang, Prog. Part. Nucl. Phys. 88, 1 (2016).

[12] K. Fukushima, D. E. Kharzeev, and H. J. Warringa, Phys. Rev. D 78, 074033 (2008).

[13] D. T. Son and A. R. Zhitnitsky, Phys. Rev. D 70, 074018 (2004).

[14] G. M. Newman and D. T. Son, Phys. Rev. D 73, 045006 (2006).

[15] Y. Burnier, D. E. Kharzeev, J. Liao, and H. U. Yee, Phys. Rev. Lett. 107, 052303 (2011).
[16] D. E. Kharzeev and H. U. Yee, Phys. Rev. D 83, 085007 (2011).

[17] D. T. Son and P. Surowka, Phys. Rev. Lett. 103, 191601 (2009).

[18] A. V. Sadofyev and M. V. Isachenkov, Phys. Lett. B 697, 404 (2011).

[19] P. V. Buividovich, Nucl. Phys. A925, 218 (2014).

[20] U. Gursoy and A. Jansen, J. High Energy Phys. 10 (2014) 92.

[21] K. Jensen, P. Kovtun, and A. Ritz, J. High Energy Phys. 10 (2013) 186.

[22] E. V. Gorbar, V. A. Miransky, I. A. Shovkovy, and X. Wang, Phys. Rev. D 88, 025025 (2013).

[23] A. Y. Alekseev, V. V. Cheianov, and J. Froehlich, Phys. Rev. Lett. 81, 3503 (1998).

[24] L. R. Baboukhadia, V. Elias, and M. D. Scadron, J. Phys. G 23, 1065 (1997).

[25] E. V. Gorbar, V. A. Miransky, and I. A. Shovkovy, Phys. Rev. C 80, 032801 (2009).

[26] E. V. Gorbar, V. A. Miransky, and I. A. Shovkovy, Phys. Rev. D 83, 085003 (2011).

[27] E. V. Gorbar, V. A. Miransky, and I. A. Shovkovy, Phys. Lett. B 695, 354 (2011).

[28] I. Amado, N. Lisker, and A. Yarom, J. High Energy Phys. 06 (2014) 084.

[29] A. Jimenez-Alba and L. Melgar, J. High Energy Phys. 10 (2014) 120.

[30] A. Yamamoto, Phys. Rev. Lett. 107, 031601 (2011).

[31] A. Yamamoto, Phys. Rev. D 84, 114504 (2011).

[32] V. Braguta, M. N. Chernodub, K. Landsteiner, M. I. Polikarpov, and M. V. Ulybyshev, Phys. Rev. D 88, 071501 (2013).

[33] V. Braguta, M. N. Chernodub, V. A. Goy, K. Landsteiner, A. V. Molochkov, and M. I. Polikarpov, Phys. Rev. D 89, 074510 (2014),

[34] J. Bloch and T. Wettig, Phys. Rev. Lett. 97, 012003 (2006).

[35] P. Hasenfratz, S. Hauswirth, T. Jörg, F. Niedermayer, and K. Holland, Nucl. Phys. B643, 280 (2002).

[36] Y. Kikukawa and A. Yamada, Nucl. Phys. B547, 413 (1999).

[37] M. Puhr and P. V. Buividovich, Comput. Phys. Commun. 208, 135 (2016).

[38] M. A. Stephanov, Phys. Rev. Lett. 76, 4472 (1996).

[39] M. Lüscher and P. Weisz, Commun. Math. Phys. 97, 59 (1985).

[40] C. Gattringer, P. E. L. Rakow, A. Schäfer, and W. Söldner, Phys. Rev. D 66, 054502 (2002).

[41] C. Gattringer, R. Hoffmann, and S. Schäfer, Phys. Rev. D 65, 094503 (2002).

[42] One of the configurations for the parameters $\beta=8.1$, $\mu=0.050$ and a magnetic flux of $\Phi_{B}=1$ caused a serious breakdown in the Lanczos algorithm when computing the overlap operator. This could not be fixed and we used only the remaining 99 configurations for this parameter set.

[43] C. Gattringer and C. B. Lang, in Quantum Chromodynamics on the Lattice. An Introductory Presentation, Lecture Notes in Physics, Vol. 788 (Springer, Berlin, 2010).

[44] M. H. Al-Hashimi and U. Wiese, Ann. Phys. (Amsterdam) 324, 343 (2009). 
[45] J. Kiskis and R. Narayanan, Phys. Rev. D 64, 117502 (2001).

[46] R. G. Edwards, U. M. Heller, J. Kiskis, and R. Narayanan, Phys. Rev. D 61, 074504 (2000).

[47] G. Basar, G. V. Dunne, and D. E. Kharzeev, Phys. Rev. D 85, 045026 (2012).

[48] T. Schaefer and E. Shuryak, Rev. Mod. Phys. 70, 323 (1998).
[49] See Supplemental Material at http://link.aps.org/ supplemental/10.1103/PhysRevLett.118.192003 for a more detailed demonstration.

[50] J. Bloch and T. Wettig, Phys. Rev. Lett. 97, 012003 (2006).

[51] I. M. Barbour, S. E. Morrison, E. G. Klepfish, J. B. Kogut, and M. P. Lombardo, Phys. Rev. D 56, 7063 (1997).

[52] T. D. Cohen, Phys. Rev. Lett. 91, 222001 (2003). 